\title{
DEHypGpOls: A Genetic Programming with Evolutionary Hyper-Parameter Optimization and its Application for Stock Market Trend Prediction
}

DAVUT ARI ( $\nabla$ dari@beu.edu.tr)

Bitlis Eren University https://orcid.org/0000-0001-6439-7957

Baris Baykant ALAGOZ

İnönü Üniversitesi: Inonu Universitesi

\section{Research Article}

Keywords: genetic programming, stock market prediction, stock price, hyper-parameter optimization, trend prediction

Posted Date: February 15th, 2022

Dol: https://doi.org/10.21203/rs.3.rs-1158075/v1

License: (c) (i) This work is licensed under a Creative Commons Attribution 4.0 International License.

Read Full License 


\title{
DEHypGpOls: A genetic programming with evolutionary hyper-parameter optimization and its application for stock market trend prediction
}

\author{
${ }^{1 *}$ Davut Ari, ${ }^{2}$ Baris Baykant Alagoz \\ ${ }^{1}$ Bitlis Eren University, Department of Computer Engineering, Bitlis, Turkey \\ ${ }^{2}$ Inonu University, Department of Computer Engineering, Malatya, Turkey \\ *Corresponding: dari@beu.edu.tr
}

\begin{abstract}
Stock markets are one of the most popular financial markets since they can bring high revenues to their investors. In order to reduce the risk factor for investors, intelligent and automated stock market trend prediction tools are developed by using computational intelligence methods. This study presents a hyper-parameter optimal Genetic Programming (GP) model generation framework for a day-ahead prediction of stock market index trends. In order to obtain the best trend prediction model from the stock market data, a Differential Evolution (DE) algorithm is implemented to optimize hyperparameters of a GP algorithm with Orthogonal Least Square (GpOls). Evolutions of GpOls agents within the hyper-parameter search space allow adaptation of GpOls algorithm for a given training dataset by enabling optimal autotuning of user-defined parameters. Authors demonstrated that this structure can significantly improve modeling performances of GpOls algorithms for data-driven modeling applications. In the study, the proposed DE based hyper GpOls (DEHypGpOls) algorithm is implemented for a day-ahead forecast problem of the Istanbul Stock Exchange 100 Index (ISE100) trends. In the experimental study, real trend data from ISE100 and seven other international stock markets are used to extract a day-ahead forecast model of daily ISE100 trends. The forecast model of the DEHypGpOls algorithm can provide $68 \%$ prediction accuracy for the test dataset. In ISE100 index market simulations, daily exchanges of ISE100 index according to buy or sell signal of the forecast model of DEHypGpOls provided about $273 \%$ more income compared to the income of a long-term investment strategy.
\end{abstract}

Keywords: genetic programming, stock market prediction, stock price, hyper-parameter optimization, trend prediction

\section{Introduction:}

Forecasting of stock market trends is one of the challenging prediction problems because several factors such as social, economical, ecological and physiological factors, even local events and trends, can affect the behavior of stock markets. For this reason, the stock markets are subject to uncertainties (Subhi Alzazah and Cheng 2020), they involve high nonlinearity, and show changing dynamics and trends(Chen et al. 2021)(Yoo et al. 2005). For this reason, in the literature, many computational intelligence methods have been used to overcome these complications. Research results have shown that automated stock market trend prediction, which is supported by computational intelligence, can reduce loss risks of the income and bring profit to the investor(Sheta et al. 2013). Some of computational methods, which have been employed to solve the stock market prediction problems, are ANN (Bisoi and Dash 2014; Akbilgic et al. 2014; Moghar and Hamiche 2020), regression models (Ananthi and Vijayakumar 2020)(Huang 2012), deep learning(Ingle and Deshmukh 2021)(Shen and Shafiq 2020), swarm intelligence (Bagheri et al. 2014), evolutionary computation (Sheta et al. 2013)(Hsu 2011)(Huang 2012), dynamical Bayesian factor graphs (Wang et al. 2015), neuro-fuzzy systems (Rajab and Sharma 2019)(Mahmud and Meesad 2016). 
A need for an initial user configuration of machine learning algorithms can reduce autonomy and adaptation skills of these algorithms. On the other hand, manual tuning of these parameters can also be an exhaustive process, and it may not be feasible in cases of existence of large amounts of data and high complexity machine learning algorithms. Real-time or online investment systems require automated and optimal modeling of financial data, and making reliable predictions for decision making and risk management algorithms. For this reason, the hyper-parameter optimization topic has been gaining more and more importance in recent years, particularly for the development of more intelligent, adaptive and autonomous systems that are capable of intelligently performing in daily life applications.

The hyper-parameter optimization tunes a set of initial user configuration parameters of machine learning algorithms so that the well-tuned machine learning algorithms can yield the best models for a given datasets(Burke et al. 2003) Thus, it contributes to performance of data-driven modeling efforts (Solomatine et al. 2009). In other words, the best practical performance of the machine learning algorithms for a given dataset can be automatically achieved by using hyper-parameter optimization techniques in practical data-driven modeling applications. In the literature, some hyper-parameter optimization techniques are based on the brute-force searching such as grid search and random search techniques (Liashchynskyi and Liashchynskyi 2019)(Villalobos-Arias and Quesada-López 2021), Also, Bayesian optimization (Cho et al. 2020), gradient-based optimization (Ollar et al. 2017), evolutionary optimization (Chatzimparmpas et al. 2021)(Schmidt et al. 2019) etc. were implemented.

Previously, genetic programming has been implemented in stock market modeling and prediction problems (Karatahansopoulos et al. 2014)(Sheta et al. 2013)(Shu-Heng Chen 2002). An enhanced tree-based GP algorithm (GpOls) was suggested with an OLS improvement (Madár et al. 2005), and it was effectively used for identification of dynamic systems (Madár et al. 2005)(Wiese 2011). However, we observed that the hyper-parameter optimization of GpOls can further improve modeling optimality and practical applicability of the algorithm in real application. Recently, the DE optimization algorithm for evolutionary hyper-parameter optimization has been discussed for several machine learning algorithms and dataset(Schmidt et al. 2019). All these progresses become a central motivation for adaptation of the DE algorithm for hyper-parameter autotuning of the GpOls algorithm in order to develop an evolutionary hyper-parameter optimal GpOls modeling framework. In the study, authors implement a DE based hyper-parameter optimal GpOls algorithm for one day-ahead forecast of the ISE100 index trends. The proposed DEHypGpOls algorithm optimizes user configuration parameters of the GpOls algorithm by means of the DE algorithm. This allows the adaptation of the GpOls modeling agents to characteristic properties of training dataset and improves performance of resulting GP forecaster models. A fine-tuned GpOls algorithm for the training data of the ISE100 dataset was obtained automatically and its evolutionary optimal forecaster model can predict a day-ahead trend in ISE100 index depending on the current trend of the ISE100 index and seven other international stock market indices. Thus, optimal data-driven modeling can be possible by using DEHypGpOls algorithm. The trend predictions of the forecast model were used to generate buy or sell signals for daily exchanges in the ISE100 index.

Organization of the paper is as follows: The following section presents a brief survey for the market prediction works in the literature. Section 2 introduces the suggested methodology for DEHypGpOls algorithm and provides preliminary knowledge for genetic programming, GpOls algorithm, differential evolution algorithms and hyper-parameter optimization. In section 3, the proposed DEHypGpOls algorithm is introduced. Section 4 presents experimental study on the ISE100 index dataset and report performance of the proposed DEHypGpOls algorithm in a day forecast of ISE 100 index. Section 5 provides a brief discussion and some concluding remarks. 


\subsection{Literature Review}

The automated stock market prediction with intelligent computation has attracted interest of many investors and researchers because a dependable knowledge of stock movements by a fraction of a second can lead to significant profits (Subhi Alzazah and Cheng 2020). Therefore, data analysis speed and prediction reliability are also major concerns in stock market prediction systems. These assets could be only achieved by several machine learning algorithms and their hybrid utilizations:

Bisoi and Dash have proposed a hybrid method that implements an infinite impulse filter based dynamic neural network (DNN) and an unscented Kalman filter for stock price prediction. (Bisoi and Dash 2014). In a another study, an efficient and interpretable neural fuzzy system was presented for stock price prediction (Rajab and Sharma 2019). To improve accuracy in predicting the next day's closing price, they selected the most effective technical indicators using Pearson's correlation coefficient in preprocessing of data (Rajab and Sharma 2019). Kumar aimed for an efficient model to predict the future price of the stock market using technical indicators that were derived from historical data. An Elman neural network was used for its ability to memorize historical information and a Gray Wolf optimization algorithm is used to optimize the ENN parameters(Kumar Chandar 2021). In another work, Yang et al. optimized neural networks in order to develop a stock market prediction model by using a Particle swarm optimization. (Yang et al. 2021). In a recent study, a hybrid model based on echo state neural network was used for prediction of stock price return volatility(Trierweiler Ribeiro et al. 2021).

A primary objective of the stock market investment is to reach a high revenue (Thakkar and Chaudhari 2021)(Gandhmal and Kumar 2019). In the classic approaches, the stock markets were modeled by using stochastic models such but the stochastic market model was not adaptive and they cannot adapt themselves for changing dynamics of the stock markets data (Lo and MacKinlay 1988). Uncertainty in local or global factors and chaotic behavior in daily investment trends cause unpredictable fluctuations of stock market indices, and this makes consistent modeling and stock market trend forecasting a difficult problem. In order to maintain the consistency of financial market models, adaptability of prediction models to changing market dynamics is necessary and evolutionary computation methods were suggested to enhance financial models (Shu-Heng Chen 2002). To benefit adaptation ability of the evolutionary computation approaches in the finance market modeling, several computational schemes were implemented in computational finance; for instance, GP algorithms, learning classifier systems, multi-objective evolutionary algorithms (MOEAs), coevolutionary optimization schemes, and evolutionary algorithms(Shu-Heng Chen 2002). Alternatively, deep neural networks have been used for stock market prediction problems in recent years (Pang et al. 2020)(Hiransha et al. 2018). Also, hybrid methods, which combine neural computation with evolutionary computation, were proposed for the market trend prediction problems (Akbilgic et al. 2014). Akbilgic et al. proposed a hybrid radial basis function neural networks with model selection by using genetic algorithms. Akbilgic et al. gave a brief discussion on the predictability of daily trend of the market index and suggested consideration of markets as evolutionary and adaptive systems than a physical or dynamic system in order to obtain more consistent prediction models (Akbilgic et al. 2014). This point motivates the current study, and to improve adaptation of GpOls algorithm for training data, hyper-parameters of GpOls algorithm are optimized by using the DE algorithm. Thus, the optimal GpOls algorithm can yield more consistent and accurate forecast models compared to non-optimal GpOls algorithms.

Previously, non-optimal GP algorithms have been implemented for solution of several financial market problems. The following work have not been performed hyper-parameter optimization for GP algorithms: GP algorithms were used for financial market modeling without (Shu-Heng Chen 2002). 
Karatahansopoulos et al. demonstrated the application of gene expression programming (GEP) for modeling the Greek index (Karatahansopoulos et al. 2014). In another stock market prediction method study, GP was implemented to detect arbitrage opportunities in the London stock market index (FTSE-100) (Markose et al. 2001). Garcia-et al. used decision trees(DT) that were generated by GP in order to detect primary financial incident changes(Garcia-Almanza and Tsang 2006). In recent work, Ari et al. discussed use of an ensemble average GP model to represent stock exchange market trend based on other international market data (Ari and Alagoz 2021).

\section{Methodology}

This study introduces a novel hyper-parameter evolutionary optimal GpOls modeling scheme in order to obtain improved data-driven prediction models. For this purpose, the DE algorithm is implemented for evolution GpOLs agents within the hyper-parameter search space of GpOls algorithm. Figure 1 depicts a general view of the proposed DEHypGpOls scheme that enables evolution of GpOls modeling agents in order to yield better models that can represent a training dataset. The DEHypGpOls algorithm performs the following stages.

(i) Differential evolution algorithm configures hyper-parameter code of GpOLs agents in hyperparameter search space.

(ii) Performances of the GpOls modeling agents are measured according to their data-driven modeling performance indices for a given training set.

(iii) DE algorithm performs hyper-parametric evolution of next generation GpOLs agents in order to increase their data-driven modeling performance in the hyper-parameter search space.

(iv) The best model from GpOLs agents population, which has the highest modeling performance, is selected as the best hyper-parameter optimal model of the DEHypGpOls algorithm.

The Best Hyper-parameter Optimal Model

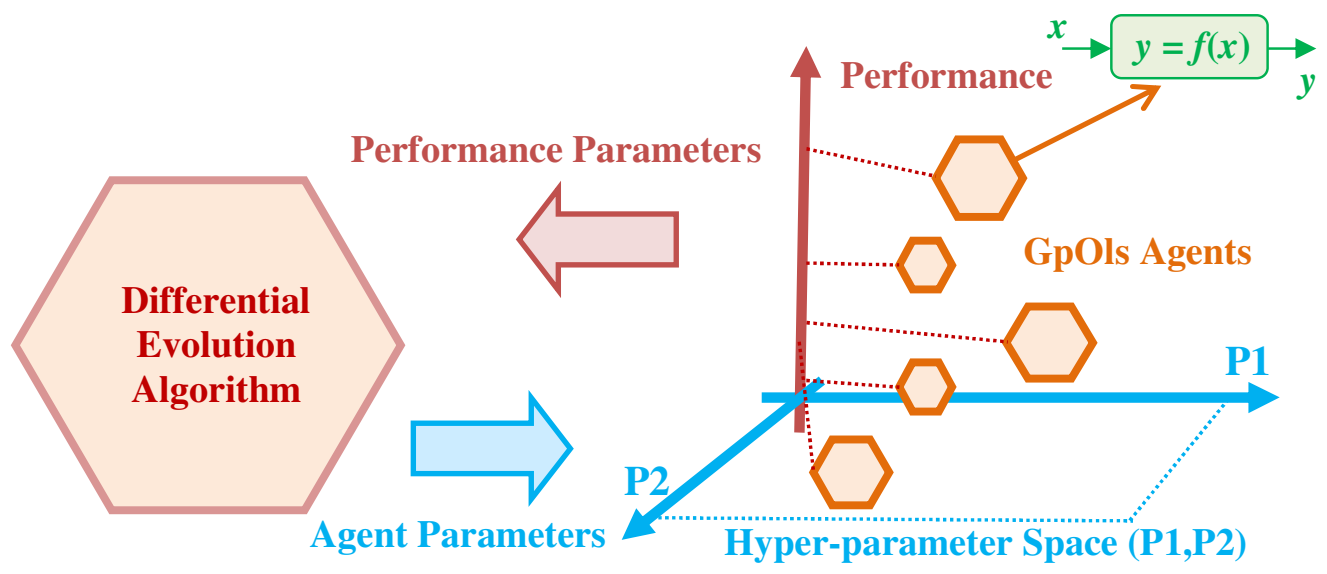

Figure 1. A schema that depicts evolution of GpOls modeling agents in the hyper-parameter search space and the best model with the highest performance

This evolutionary optimization process optimizes a hyper-parameter code that expresses user-defined parameters of GpOls agents. This process autonomously improves the modeling performance without a need of expert configuration on the GpOls algorithm. This perspective contributes to development of autonomous data-driven modeling algorithms for real-time applications. 


\subsection{Genetic Programming Preliminaries and GpOls Algorithm}

GP is an evolutionary computation (EC) methodology that can automatically formulate an optimal solution of modeling, design and computer programming problems (Koza 1992). The GP has become a popular EC technique because of its success in applications and its potentials to generate effective automatic modeling solutions for problems from many science and engineering fields (López-López et al. 2019)(Koza et al. 2004) (Dal Piccol Sotto and De Melo 2014). In GP literature, the automatic problem solving ability of GP techniques is widely utilized in symbolic regression problems for datadriven modeling works (Dal Piccol Sotto and De Melo 2014). The GP method has been used extensively in many application areas, for example production scheduling (Nguyen et al. 2017), optimal water reservoir-operating (Ashofteh et al. 2015), energy of residential buildings (Castelli et al. 2015; Kaboli et al. 2017; Tahmassebi and Gandomi 2018), educational technologies (Zafra and Ventura 2012), urban planning (Patnaik and Bhuyan 2016), geotechnical design (Keshavarz and Mehramiri 2015), hydrology (Shoaib et al. 2015), medicine (De Falco et al. 2018). Also, GP has been widely utilized in many computer science problems such as classification problems (Tran et al. 2016)(Kuo et al. 2007), computer vision (Liu et al. 2016), image processing (Shao et al. 2014)(Liang et al. 2020), signal processing (Feli and Abdali-Mohammadi 2019), artificial neural network design (Suganuma et al. 2017). Moreover, GP methods were used in the field of evolutionary hardware (Mora et al. 2019) and circuit design (Sikulova et al. 2014)(Koza et al. 2004). One can find many application of GP in the field of economy (e.g., financial fraud detection (Li and Wong 2015) and green supplier selection (Fallahpour et al. 2016) )

FigureFigure 2 shows an example syntax tree of a GP model. This tree is a graphical expression of the population individual that represents a candidate solution model $y=\left(x_{1}+x_{2}\right)-\left(x_{1}+x_{3}\right) \times x_{2}$. The genetic processes such as crossover, mutation produce new GP syntax trees, and those better fitting to solution of the problem, are selected to compose new generations of GP population individuals. During the repeating cycles of genetic processes, the syntax trees are evolved towards better fitting trees, and they express more suitable candidate solutions for a problem.

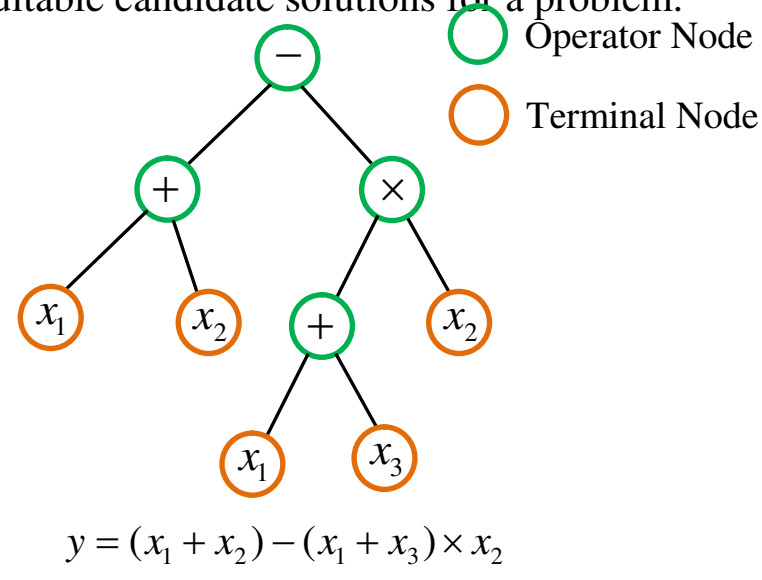

Figure2. An example GP tree structure that represents $y=\left(x_{1}+x_{2}\right)-\left(x_{1}+x_{3}\right) \times x_{2}$ solution formulation for a regression problem

GpOls algorithm is a tree-based GP algorithm that involves Orthogonal Least Square (OLS) calculation. The OLS adaption to GP algorithm improves fitting of the nonlinear mathematical model 
to the training dataset. The GpOls algorithm was proposed for the identification of nonlinear inputoutput models by implementing a tree-based genetic programming technique (Madár et al. 2005). It was used for the model identification in control application (Wiese 2011). A major advantage of GpOls algorithm is the production of accurate and interpretable models (Madár et al. 2005). Essentially, it implements an orthogonal least square algorithm to estimate the contribution of each nonlinear modeling subtrees to a resulting linear-in model and thus yields a robust and interpretable models in the form of weighed sum of selected nonlinear subtree models (Madár et al. 2005). The linear-in model of GpOls algorithm was formulated in the form of

$$
y(k)=\sum_{i=1}^{M} p_{i} F_{i}(x(k)),
$$

where $F_{i}(x(k))$ stands for the nonlinear functions or terminals that are obtained from subtrees of nonlinear model fragments and terminal nodes (Madár et al. 2005). The parameter $p_{i}$ is weights of subtree models. Input vector of this linear-in model was written by

$$
x(k)=\left[x_{1}(k) x_{2}(k) \ldots x_{n}(k)\right] .
$$

This methods commonly use an operator set that involves linear operators, i.e., $\{+,-\}$ and nonlinear operators, i.e., $\{x, /\}$. In operation, the GoOls algorithm initially decomposes GP syntax tree into the subtrees of nonlinear model fragments that are initialized with nonlinear operator nodes or a terminal node as described in Figure 3. Each of these subtrees forms a nonlinear function or a terminal that is denoted by $F_{i}(x(k))$ in the linear-in model formulation (Equation (1)). Figure 3 shows an example decomposition of the syntax tree (in Figure 1) into subtrees of the nonlinear functions to compose a linear-in model. Whenever the root node is a nonlinear operator or a terminal, it forms an element of the function set $F_{i}(x(k))$ to consider in equation (1) (Madár et al. 2005).

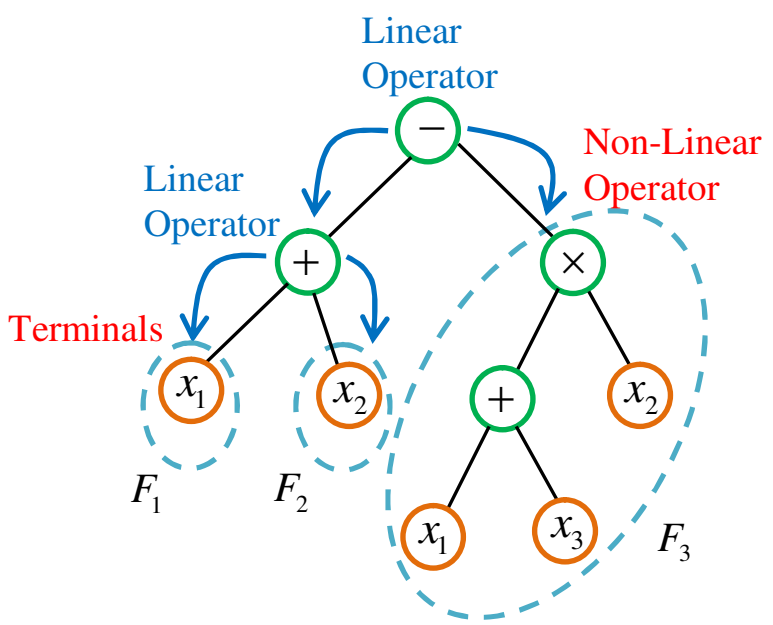

Figure 3. Decomposition of the syntax tree into nonlinear functions $F_{1}, F_{2}$ and $F_{3}$

In Figure 3, the selected subtrees produce the functions $F_{1}=x_{1}, F_{2}=x_{2}$ and $F_{2}=\left(x_{1}+x_{3}\right) \times x_{2}$. Then, the linear-in model can be written by

$$
\begin{aligned}
& y(k)=\sum_{i=1}^{M} p_{i} F_{i}=p_{1} F_{1}+p_{2} F_{2}+p_{3} F_{3}, \\
& =p_{1} x_{1}+p_{2} x_{2}+p_{3}\left(\left(x_{1}+x_{3}\right) \times x_{2}\right)
\end{aligned}
$$


To calculate $p_{i}$ weight coefficients, the least square method was used to fit a training dataset that can expressed in the form of a set of $\mathrm{k}+1$-tuble for a $\mathrm{k}$ input-one output model as $T=\left\{\left(x_{1}(1) x_{2}(1), x_{k}(1), y(1)\right),\left(x_{1}(2) x_{2}(2), x_{k}(2), y(2)\right), . .,\left(x_{1}(n) x_{2}(n), x_{k}(n), y(n)\right)\right\}$. The equation (3) was written in the vector form for whole training data set as (Madár et al. 2005)

$$
y=F p+e
$$

where the matrix $F$ denotes the regression matrix, the parameter $p$ is a vector of weights $p_{i}$ and $e$ represents error vector for this regression problem. It is solved by an orthogonal least square technique that transforms the columns of the F matrix into a set of orthogonal basis vectors (Madár et al. 2005).

\subsection{Differential Evolution}

Differential Evolution (DE) algorithm is an effective evolutionary optimization method that was developed by Storn and Price (Storn and Price 1995, 1997)(Cui et al. 2018). The DE algorithm is a popular metaheuristics method that can iteratively improve candidate solutions for an optimization problem by considering fitness values of a predefined objective function. It implements essential genetic processes (mutation, crossover and selection) by using relatively simple formulas to form a new generation of candidate solutions and maintain the best candidates through generations by selecting them according to their fitness performance. A brief introduction of the DE algorithm was summarized as follows (Qin et al. 2009):

A candidate solution of the DE algorithm is expressed in D-dimensional parameter space in the form of

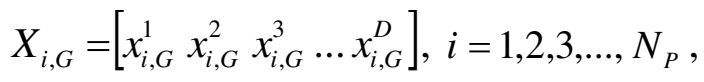

where the subscript $G$ represents the generation number and the parameter $N_{p}$ is the population size of the candidate solution set. The D-dimensional parameter space forms a search space of the algorithm to find out an optimal solution. At the initialization stage of DE algorithm, the individuals of the population are randomly distributed into the search space that are confined by the boundaries $X_{\min }=\left[\begin{array}{lllll}x_{\min }^{1} & x_{\min }^{2} & x_{\min }^{3} & \ldots & x_{\min }^{D}\end{array}\right]$ and $X_{\max }=\left[\begin{array}{llll}x_{\max }^{1} & x_{\max }^{2} & x_{\max }^{3} \ldots x_{\max }^{D}\end{array}\right]$ (Qin et al. 2009). At each iteration of the DE algorithm, evolutionary processes i.e., mutation, crossover and selection processes are employed to obtain new generation candidate solutions, and the group of the candidate solutions is selected according to their fitness values in order to maintain generations with better fitting solutions. Fitness values are calculated according to a predefined objective function of the optimization problem. As a result of repeated improvement of generation based on evolutionary processes, the candidate solutions evolve towards the optimum solutions that are defined by the objective function (Qin et al. 2009).

To form an initial population of candidate solutions, candidate solutions are distributed uniformly into the entire search space by using uniform random numbers within the search ranges that are bounded by predefined upper and lower boundaries $X_{\min }=\left[\begin{array}{llll}x_{\min }^{1} & x_{\min }^{2} & x_{\min }^{3} \ldots x_{\min }^{D}\end{array}\right]$ and $X_{\max }=\left[\begin{array}{llll}x_{\max }^{1} & x_{\max }^{2} & x_{\max }^{3} \ldots x_{\max }^{D}\end{array}\right]$ (Qin et al. 2009). In the current study, the following formulations of DE algorithm are implemented for the genetic processing:

(i) Mutation Process: The mutation process forms new candidate solutions of the population by performing geometrical deviations of solutions in the search space. The new candidate solutions, 
which are represented by the vector set $\mathrm{V}_{\mathrm{i}, \mathrm{G}}=\left\{v_{i, G}^{1}, v_{i, G}^{2}, v_{i, G}^{3}, \ldots, v_{i, G}^{D}\right\}$, are formed in the search space. Several mutation strategies have been suggested in the literature. We implemented the "DE/rand/1" strategy that formulated the mutation as follows (Qin et al. 2009).

$$
\mathrm{V}_{\mathrm{i}, \mathrm{G}}=X_{r_{1}^{i}, G}+S .\left(X_{r_{2}^{i}, G}-X_{r_{3}^{i}, G}\right)
$$

where the subscripts $r_{1}^{i}, r_{2}^{i}, r_{3}^{i}$ are randomly selected numbers of individuals from the population in the range of $\left[1, N_{p}\right]$. The parameter $S$ is the scale factor that is used to adjust length of the difference vector $\left(X_{r_{2}^{i}, G}-X_{r_{3}^{i}, G}\right)$ (Qin et al. 2009). The scaled difference vector determines the range of alterations in the mutation process of solutions.

(ii) Crossover Process: After the mutation process, a crossover operation is performed with a probability rate of $C_{r}$ to form new individuals of the population (Qin et al. 2009). The new candidate solution of the crossover process are represented by the vector set $\mathrm{U}_{\mathrm{i}, \mathrm{G}}=\left\{u_{i, G}^{1}, u_{i, G}^{2}, u_{i, G}^{3}, \ldots, u_{i, G}^{D}\right\}$, The parameter $C_{r}$ is the crossover rate that is used to adjust the frequency of crossover operations and new candidate solutions $\mathrm{U}_{\mathrm{i}, \mathrm{G}}=\left\{u_{i, G}^{1}, u_{i, G}^{2}, u_{i, G}^{3}, \ldots, u_{i, G}^{D}\right\}$ are formed by randomly selected part of a candidate solution of mutated solution $\mathrm{V}_{\mathrm{i}, \mathrm{G}}$. We implemented the "DE/rand/1" strategy that formulated the crossover process as follows (Qin et al. 2009).

$$
u_{i, G}^{j}=\left\{\begin{array}{lc}
v_{i, G}^{j} & \text { if }\left(\operatorname{rand}_{j}(0,1) \leq C_{r}\right) \text { or }\left(j=j_{\text {rand }}\right) \\
x_{\mathrm{i}, \mathrm{G}}^{j} & \text { else }
\end{array}, j=1,2,3, \ldots, D\right.
$$

(iii) Selection Process: The selection process is an essential process of differential evolution that allows the maintenance of good candidate solutions through generations of evolution process. The objective function value of new individuals and old individuals are denoted by $f\left(U_{i, G}\right)$ and $f\left(X_{i, G}\right)$ respectively, and the best fitting individuals for a minimization problem are selected according to objective function values as follows (Qin et al. 2009).

$$
X_{i, G+1}=\left\{\begin{array}{cc}
U_{i, G} & \text { if } f\left(U_{i, G}\right) \leq f\left(X_{i, G}\right) \\
X_{\mathrm{i}, \mathrm{G}} & \text { else }
\end{array}\right.
$$

\subsection{Hyper-parameter optimization}

Hyper-parameters optimization techniques aim to tune hyper-parameters of the machine learning algorithm in order to obtain the best machine learning model for a given dataset. In general, the fitting performance of models for the dataset is used to evaluate the optimality of the tuned hyperparameters, and the hyper-parameter optimization achieves the parametrical optimality of machine learning tools for the training dataset, and it contributes to the practical performance of data-driven modeling application by auto-tuning the user-defined parameters of algorithms. An important requirement for the optimization techniques, which are used in the hyper-parameter optimization task, is fast convergence to optimal points. Otherwise, in cases, where machine learning algorithms have high computational load or training dataset is too large or big data, the hyper-parameter optimization task leads to severe increase of the computational load in the data-driven modeling task and becomes an impractical task for real-world applications.

The well-tuned machine learning algorithms yield the best models for a given datasets(Burke et al. 2003). There are generally two tuning methods for user-defined parameters of the machine learning 
methods. These are manual-tuning and automatic-tuning of parameters (Wu et al. 2019). In some cases, it is quite difficult to manually set the optimum parameter value in computational intelligence methods (Trujillo et al. 2020). In a data-driven intelligent system point of view, the hyper-parameters of machine learning algorithms should be automatically tuned without a need for the expert knowledge on the dataset and/or manual configuration on the machine learning algorithms. Besides the requirement of autonomous configuration algorithms in data-driven modeling tasks, when the amount of data is quite high (e.g, big data) or a live data stream is used for real-time modeling, automatic-tuning, namely hyper-parameter optimization of algorithms, is a necessity in online realworld applications. Therefore, recent works have revealed the fact that automatic machine learning (AutoML) and adaptive deep neural networks required the optimal tuning of numerous algorithm parameters (Feurer and Hutter 2019). In general, hyper-parameter optimization offers the following plus points (Feurer and Hutter 2019):

- System Autonomy and Adaptivity: The machine learning tasks require less human efforts or expertise when hyper-parameters of machine learning tools are automatically configured.

- Optimal System Performance: Parametric optimality of machine learning algorithms improves intelligent system performances.

- Practical Effectiveness: Hyper-parameter optimization maximizes the benefits and applicability of machine learning algorithms in real-world applications.

In the current work, authors have preferred an evolutionary hyper-parameter optimization technique to increase the accuracy of GP based stock market trend prediction models. In the previous work, authors demonstrated extracting a regression model of ISE100 index stock market by using ensemble average GP modeling method(Ari and Alagoz 2021). However, this approach cannot yield effective modeling for solutions of the day-ahead forecast problem. The main reason is that user parameters of a GP algorithm are manually configured for a limited number of set and trial efforts, and it does not ensure the hyper-parameter optimality of the GP algorithm for the training dataset. In the current study, to alleviate this shortcoming, we proposed an evolutionary hyper-parameter optimal GP algorithm, namely the DEHypGpOls algorithm.

\section{DEHypGpOls: An Evolutionary Optimization Scheme for hyper-parameters optimal GpOls Algorithm}

The main focus of this study is to generate a day ahead forecast model that can predict the next-day behavior of the ISE 100 index. Our tests showed that modeling performance of the GpOls algorithm depends on the configuration of hyper-parameters of the algorithm. Therefore, authors adopted a DE algorithm to obtain evolutionary optimal-tuned GpOls programming agents. Accordingly, the GpOls algorithm itself is considered as programming agents that evolve in the search space according to a set of hyper-parameter code. The DE algorithm improves the hyper-parameters of the GpOls agents according to their data-driven modeling performance. Thus, the GpOls programming agents are evolved to the best hyper-parameter configuration that yields the best fitting model for a day ahead forecast problem of ISE100 index trend. In this way, the proposed evolutionary hyper-parameter optimization minimizes human intervention and allows reaching an optimal model generation performance of the GpOls algorithm. Such evolutionary hyper-parameter optimization modification improves

(i) autonomy of the GpOls algorithm by adapting initial settings of the algorithm according to characteristic properties of data and modeling problem, 
(ii) model generation performance by selecting the best model from evolving GpOLs agents,

(iii) practical effectiveness of GpOls by reducing the need for configuring by a human expert.

Figure 4 shows a flow chart of the proposed DEHypGpOls method. According to this flow chart, each GpOls modeling agent is evolved to produce a model with a better Mean Square Error (MSE) performance. After completion of differential evolution of GP agents, the GP agents provide the best model, which fits to the training dataset with the lowest MSE. Then, the performance evaluation of the best model is carried out in a simulation environment by implementing a test data set.

According to the flow chart in Figure 4, steps of the proposed DEHypGpOls algorithm can be written as follows:

Step 1: Generate randomly initial hyper-parameter solutions by using the DE algorithm.

Step 2: Run GpOls Modeling agents and obtain data-driven models by using training dataset and evaluate MSE performance of these models according to the training dataset.

Step 3: Produce next generation hyper-parameter solutions by applying genetic process (mutation, crossover and selection) of the DE algorithm.

Step 4: Select hyper-parameter solutions that have lower MSE values and form a set of hyperparameter solutions that better represents the training dataset.

Step 5: If the stopping criteria is not met, turn to Step 2. Otherwise, select the best model with the lowest MSE value and stop the program.

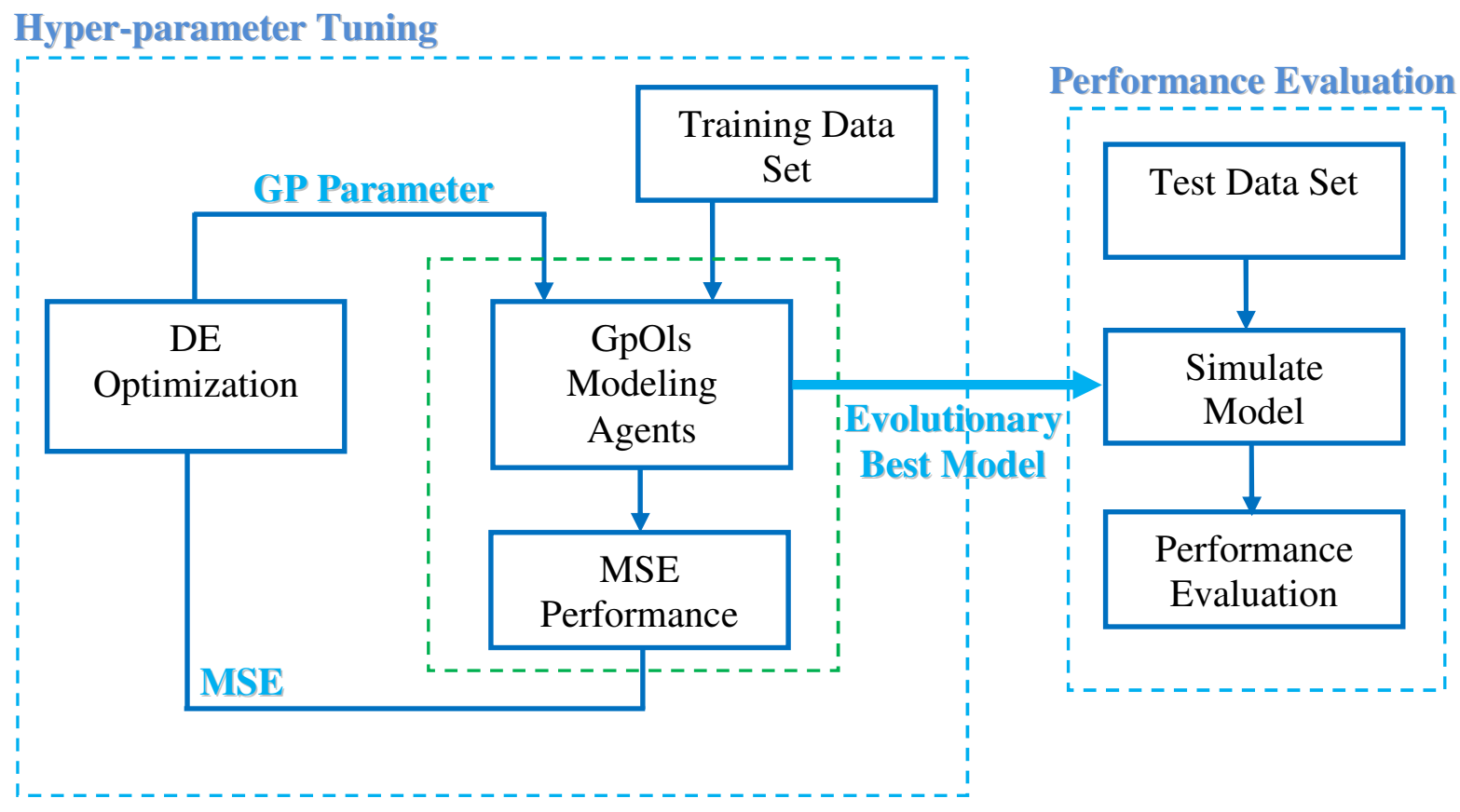

Figure 4. A flow chart of the proposed DEHypGpOls algorithm

In the stock market trend prediction application, a day ahead forecast of the ISE100 index trend is performed by using the current day trends of the ISE100 index and the other 7 world stock markets. Since stock markets are highly dynamic, several unpredictable factors and uncertainty are effective, forecast models can represent market behavior for a limited time horizon, and they need adaptation to 
up-to-date conditions of market trends. To keep the model up-to-date, $\mathrm{P}$ numbers of previous days' data are used to compose a training data set to generate a prediction model. The $\mathrm{P}$ numbers of previous days are called the modeling horizon. Test dataset is composed with L numbers of following days' data. The L numbers of the following days in near future are called forecast horizon. Figure 5 shows a diagram for this finite time-horizon modeling and forecasting scheme. Performance of the best model is evaluated according to a test dataset that is not involved in the training process. This model generation approach keeps the forecast model up-to-date with data of the most recent $\mathrm{P}$ day period and enables consideration of the latest $\mathrm{P}$ day changing market dynamics in the forecaster model. A similar finite time-horizon solution has been implemented in the model predictive control since the 1980s. Current response of the control system is optimized by considering future time slots that are called prediction horizon (Kwon et al. 1982)(García et al. 1989) and it is a very effective method for optimality of control system responses in changing environments and conditions. Similar approaches are also used for neural networks to keep the neural model up-to-date for changing training dataset, for instance, progressive neural network(Gideon et al. 2017), transfer learning(Zhuang et al. 2021).

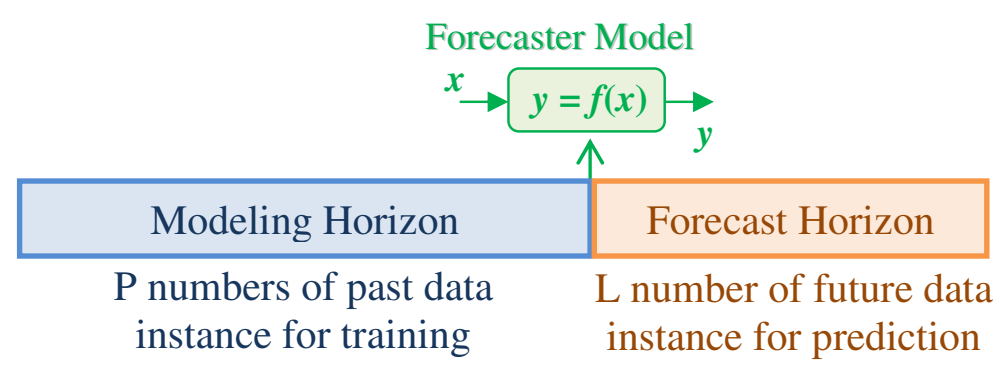

Figure 5. A diagram for the finite time-horizon modeling approach

\section{Experimental Study}

\subsection{Experimental Setup and Software Tools}

Experimental study was conducted in the Matlab environment. A Matlab tool, which combines DE algorithm and GpOls algorithm, was developed and it was used to obtain the evolutionary optimal forecaster model. Then, the performance evaluation of models was performed by using the test dataset and stock market simulation model in the Matlab. All experiments were conducted by using a PC that has a four-core Intel(R) Core(TM) i7-5700HQ CPU 2.70GHz and 16 GB RAM.

The GpOls is an effective software tool that was developed to generate polynomial regression models in the Matlab environment by using a tree-based GP algorithm with an OLS improvement (Madár et

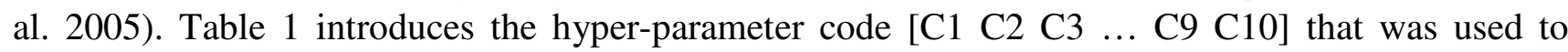
optimize of GpOls agents by the DE algorithm. These hyper-parameters can affect modeling performance of GpOls toolbox (Madár et al. 2005)(Wiese 2011) The Matlab DE tool, which was provided by Mostapha Kalami Heris from Yarpiz, was modified to optimize the hyper-parameter code of GpOls agents. 
Table 1. Hyper-parameter code of GpOLs agents that was optimized by the DE algorithm

\begin{tabular}{lcc}
\hline \multicolumn{1}{c}{ GpOls Parameters } & Value Ranges & $\begin{array}{c}\text { Hyper-parameter } \\
\text { Code }\end{array}$ \\
\hline Generation Gap & $\{0,1\}$ & $\mathrm{C} 1$ \\
Probability for Crossover & $\{0,1\}$ & $\mathrm{C} 2$ \\
Probability for Mutation & $\{0,1\}$ & $\mathrm{C} 3$ \\
Selection Type & {$[0,1,2 \ldots$ Population size $]$} & $\mathrm{C} 4$ \\
One or Two Point Crossover & {$[1,2]$} & $\mathrm{C} 5$ \\
Tree Size Penalty: Weighting & $\{0,1\}$ & $\mathrm{C} 6$ \\
Tree Size Penalty: Location & {$[0,1,2 \ldots$ Max Tree Size $]$} & $\mathrm{C} 7$ \\
OLS Tree Pruning Threshold & $\{0,1\}$ & $\mathrm{C} 8$ \\
Polynomial Evaluation on/off & {$[0,1]$} & $\mathrm{C} 9$ \\
Evaluate all or only new individuals & $0=$ new; $1=$ all & $\mathrm{C} 10$
\end{tabular}

\subsection{Experimental Dataset}

Istanbul Stock Exchange (ISE100) dataset (Akbilgic et al. 2014) was used in this experimental study. Table 2 introduces the dataset and the related model parameters. By using these data, a day ahead forecast model of the ISE100 index trend was obtained by using the proposed DEHypGpOls algorithm. The next day ISE100 index trend forecast $\left(y_{n+1}\right)$ is expressed as a function of the current day SP $\left(x_{1, n}\right), \operatorname{DAX}\left(x_{2, n}\right), \operatorname{FTSE}\left(x_{3, n}\right), \operatorname{NIK}\left(x_{4, n}\right), \operatorname{BVSP}\left(x_{5, n}\right), \operatorname{EU}\left(x_{6, n}\right), \operatorname{EM}\left(x_{7, n}\right)$ and $\operatorname{ISE} 100$ index trends $\left(y_{n}\right)$, which can be expressed as follows:

$$
y_{n+1}=f\left(x_{1 n}, x_{2 n}, x_{3 n}, x_{4 n}, x_{5 n}, x_{6 n}, x_{7 n}, y_{n}\right)
$$

Table 2. Istanbul Stock Exchange (ISE100) Dataset(Akbilgic et al. 2014)and model parameters

\begin{tabular}{clcc}
\hline Stock Markets & \multicolumn{1}{c}{ Explanations } & Parameters & Type \\
\hline ISE100 & $\begin{array}{l}\text { Istanbul stock exchange national 100 index } \\
\text { trend }\end{array}$ & $y_{n}$ & Input \\
SP & Standard \& poor's 500 return index trend & $x_{1, n}$ & Input \\
DAX & $\begin{array}{l}\text { Stock market return index trend of } \\
\text { Germany }\end{array}$ & $x_{2, n}$ & Input \\
FTSE & Stock market return index trend of UK & $x_{3, n}$ & Input \\
BVSP & Stock market return index trend of Japan & $x_{4, n}$ & Input \\
EU & Stock market return index trend of Brazil & $x_{5, n}$ & Input \\
EM & MSCI European index trend & $x_{6, n}$ & Input \\
MSCI emerging markets index trend & $x_{7, n}$ & Input \\
Next day ISE100 & $\begin{array}{l}\text { Istanbul stock exchange national 100 index } \\
\text { trend }\end{array}$ & $y_{n+1}$ & Output
\end{tabular}


Figure 6 depicts a schematic ofan active stock market index forecast system that is composed of a database for hosting market data, DEHypGpOls hyper parameter tuner and an evolutionary optimal forecaster model.

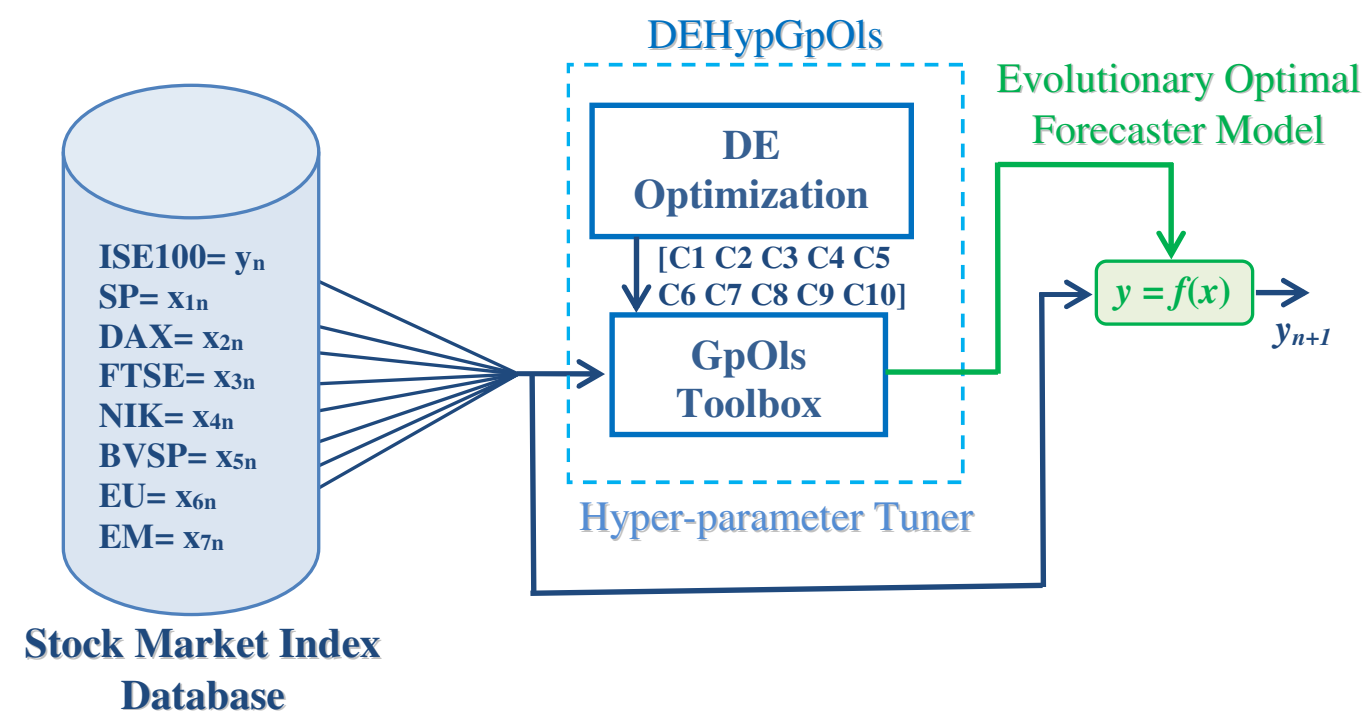

Figure 6.A schematic of an active stock market index forecaster that implements the DEHypGpOls algorithm

\subsection{Experimental Results}

In the experimental study, the past 335 days index trends of Stock Exchange (ISE100) Dataset were used to compose a training dataset to generate an evolutionary optimal forecaster model (a modeling horizon with $\mathrm{P}=335)$, and this model was used to predict the following day ISE100 index trends for next 200 days. (a forecast horizon with $\mathrm{L}=200$ ). The DEHypGpOls algorithm produced a day ahead forecast model of ISE100 index trend as

$$
y_{n+1}=0.19683 *\left(\mathrm{x}_{1, \mathrm{n}}-\left(\left(\mathrm{x}_{6, \mathrm{n}}-\left(\left(\left(\left(\mathrm{y}_{\mathrm{n}}-\mathrm{x}_{6, \mathrm{n}}\right)+\left(\mathrm{x}_{5, \mathrm{n}}+\mathrm{x}_{5, \mathrm{n}}\right)\right)-\mathrm{x}_{7, \mathrm{n}}\right)-\mathrm{x}_{7, \mathrm{n}}\right)\right)-\mathrm{x}_{1, \mathrm{n}}\right)\right)+1.56828 .10^{-3}
$$

Figure 7 shows decrease of MSE for the best GpOls agents when optimizing hyper-parameters of the GpOls agents. This decrease in MSE validates the adaptation of GpOls algorithm according to the training dataset. The MSE performance of the best GpOls agent decreased to a value of 3.1285e-04. The resulting evolutionary optimal model is given by equation (10) provided a prediction accuracy value of $68 \%$ for a forecast horizon of 200 days. 


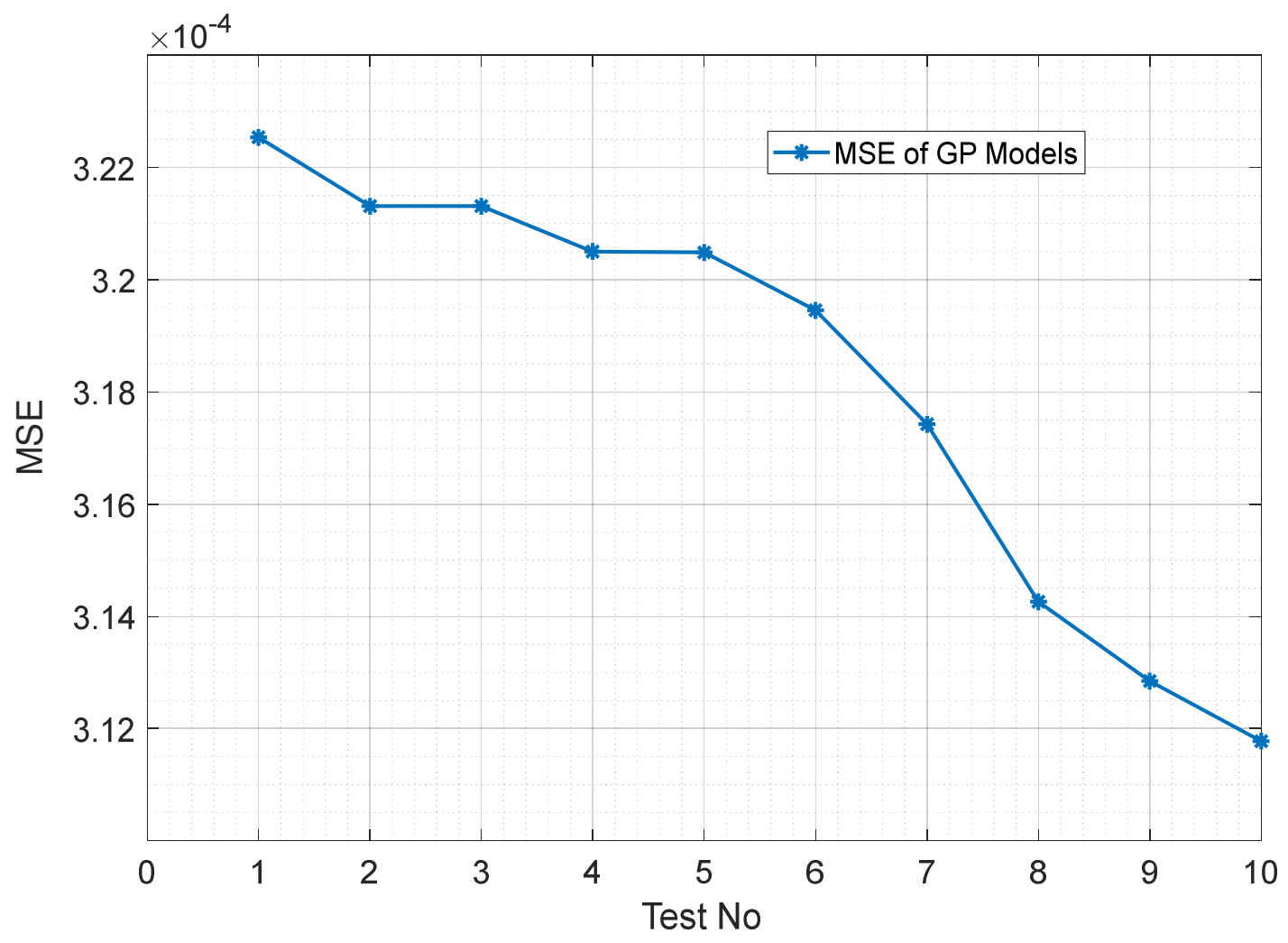

Figure 7.Optimization of hyper-parameters of GpOls agents

To evaluate the prediction performances of the evolutionary optimal model (equation (10)) in forecasting horizon, the mean square error (MSE), the mean absolute error (MAE), relative absolute error (RAE) and root mean square error (RMSE) are used:

$$
\begin{aligned}
& M S E=\frac{1}{L} \sum_{n=0}^{L-1}\left(y_{d, n}-y_{n+1}\right)^{2} \\
& M A E=\frac{1}{L} \sum_{n=0}^{L-1}\left|y_{d, n}-y_{n+1}\right| \\
& R A E=\frac{1}{L} \sum_{n=0}^{L-1} \frac{\left|y_{d, n}-y_{n+1}\right|}{\left|y_{d, n}\right|} \\
& R M S E=\sqrt{M S E}
\end{aligned}
$$

where the parameter $y_{d, n}$ is actual value of ISE100 index trend within forecasting horizon (ground truth data) and the parameter $y_{n+1}$ denotes the day ahead forecasts of the optimal forecaster model. To analyze investment performance, a stock exchange market investment simulation was carried out by using Istanbul Stock Exchange (ISE100) Dataset (Akbilgic et al. 2014). In this simulation, the automated daily investment of the ISE100 index was performed according to the buy or sell signal of the optimal forecaster models of DEHypGpOls algorithm. The buy or sell signal is generated according to the sign of trend predictions as follows:

$$
B S=\left\{\begin{array}{cc}
1 & y_{n+1} \geq 0 \\
-1 & y_{n+1}<0
\end{array},\right.
$$


where $B S=1$ is a buy signal and $B S=-1$ is a sell signal. Figure 8(a) shows actual ISE100 index trend values $\left(y_{d, n+1}\right)$ and the forecasted ISE100 index values $\left(y_{n+1}\right)$ and Figure 8(b) shows corresponding buy and sell signals that were generated according to equation (15). Figure 9 shows true buy-sell recommendation instances with a value of 1 and false buy-sell recommendation instances with a value of 0 . There are several periods where consecutive daily true investment can be performed in the simulations. False investments are mainly short-term and they are followed by true investment days. Thus, incomes in Figure 10 grow steadily.
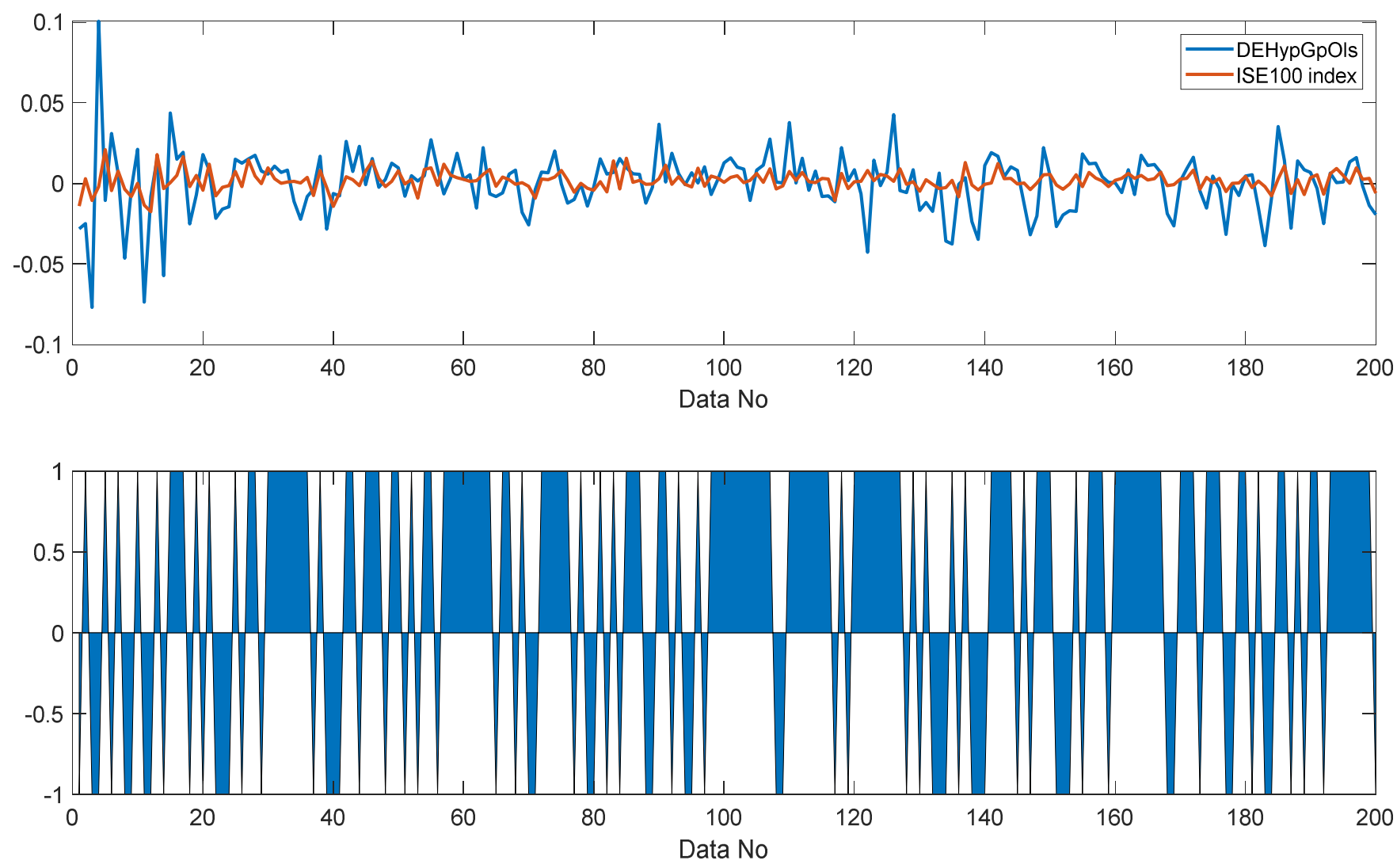

Figure 8. (a) Actual ISE100 index trend values $\left(y_{d, n}\right)$ and the predicted ISE100 index values $\left(y_{n+1}\right)$, (b) Corresponding buy signal (1) and sell signal (-1).

Daily profits of the automated daily investment system that uses buy-sell signals from a day ahead forecast model of DEHypGpOls algorithm (equation (10)) and a day ahead forecaster model of ANN were compared with the income of a long-term investment strategy in the stock exchange market simulation. Figure 10 shows daily income curves from the simulations. In these simulations, daily investments according to the evolutionary optimal forecaster of DEHypGpOls algorithm earned about $3.7552 \$$ per $1 \$$ in 200 days. In the same simulation, the daily investment according to a neural forecaster with ANN earned about 2.5797 \$ per 1 \$. A long-term investment strategy earned 1.0162 per $1 \$$ after 200 days. Simulation result indicated that the forecaster with the DEHypGpOls algorithm can provide about $273 \%$ more income compared to the income of a long-term investment and the neural forecaster can provide about $152 \%$ more income compared to the income of a longterm investment strategy. 


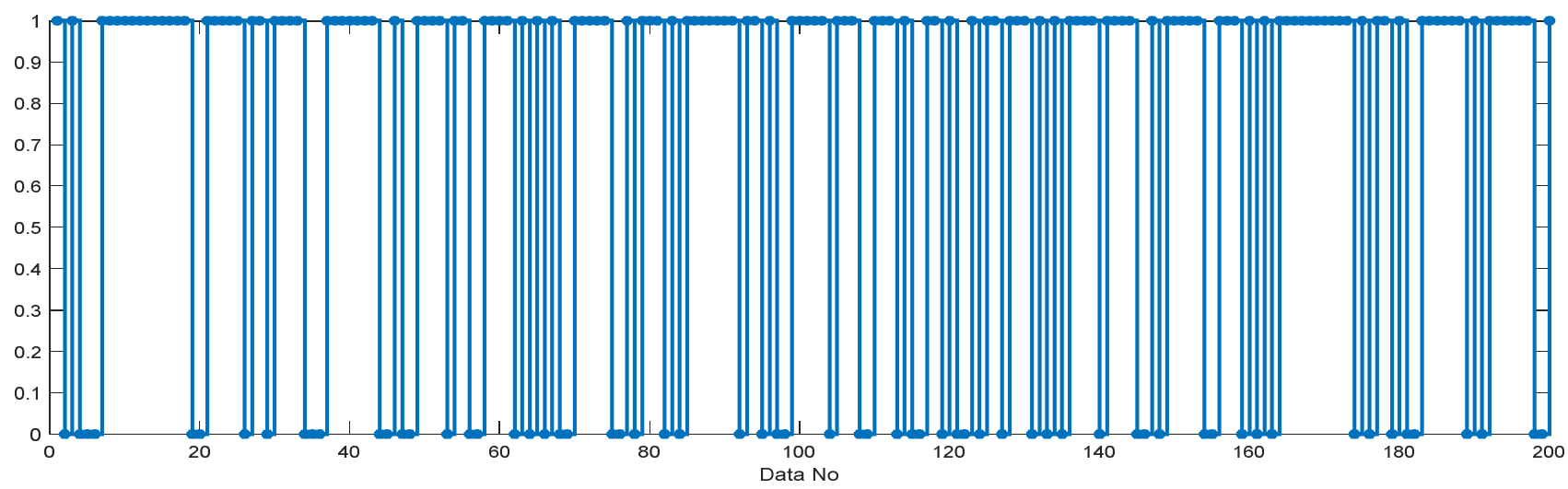

Figure 9. Buy-sell recommendation status: a value of 1 for true buy-sell recommendation instances and a value of 0 for false buy-sell recommendation instances

The accuracy of buy-sell recommendation was calculated by using true buy signal (TB), false buy signal (FB), true sell signal (TS) and false sell signal (TS) as follows

$$
\text { Accurarcy }=\frac{T B+T S}{T B+F B+T S+F S}
$$

Table 3 shows recommendation accuracy of various day-ahead forecaster models.

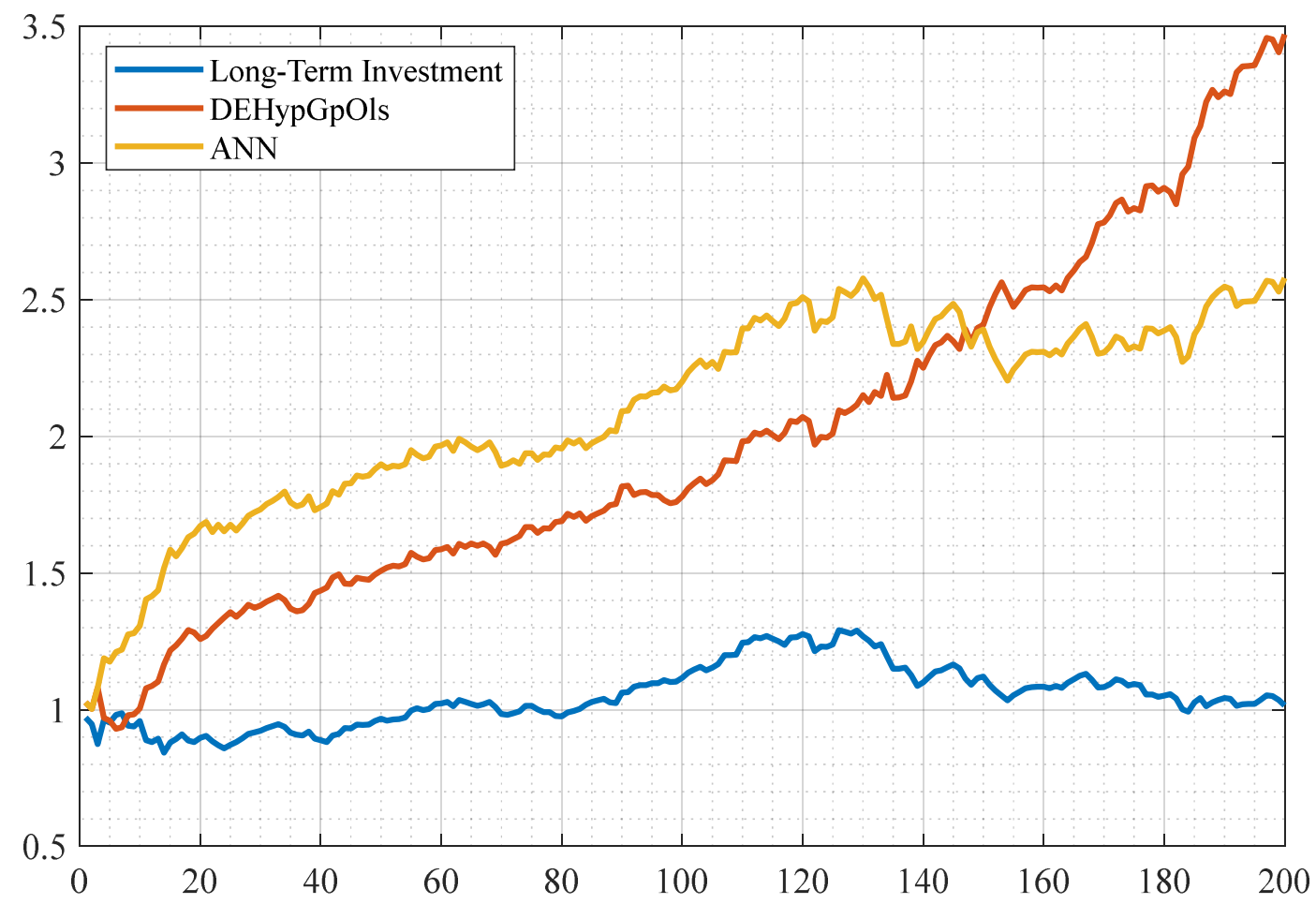

Figure 10. Daily income curves from the stock exchange market simulation for a long-term investment strategy, daily investments based on an active stock market index forecaster that implements the a day-ahead forecast models of the DEHypGpOls algorithm and the ANN. 
Table 3.Recommendation accuracy of various a day-ahead forecast models

\begin{tabular}{cc}
\hline Methods & $\begin{array}{c}\text { Recommendation } \\
\text { Accuracy }(\%)\end{array}$ \\
\hline Proposed Method & 68 \\
ANN & 65 \\
HRBF-NN (Akbilgic et al. 2014) & 65
\end{tabular}

Table 4. Performance comparisons of a day-head forecast models

\begin{tabular}{ccc}
\hline Performance Index & Proposed Method & ANN \\
\hline MSE & 0.000313 & 0.000334 \\
MAE & 0.012395 & 0.012804 \\
RAE & 1.617475 & 1.682574 \\
RMSE & 0.017687 & 0.018281 \\
Accuracy & 0.68 & 0.65 \\
Final income for 1 \$ investment after & 3.7552 & 2.5797
\end{tabular}

200 days

\section{Conclusions}

Autonomous stock market forecast tools are helpful to reduce investment risks and possibility of increasing investment revenue. Performance of stock market forecast tools is a vital problem when considered highly uncertain and short-term market dynamics in the market trends. This study introduced a new hyper-parameter optimal genetic programming based data-driven model generation framework for a day-ahead prediction of ISE100 index trends. The prediction model of the DEHypGpOls algorithm can provided $\% 68$ prediction accuracy in a forecast horizon of 200 days and the market investment simulation on the ISE100 index dataset clearly demonstrated that the active stock market index forecaster system that implements the evolutionary optimal model of DEHypGpOls algorithm can provided about $273 \%$ more income than a long-term investment player in a 200 days market simulation. Some remarks of the study can be summarized as

* The hyper-parameter optimization can significantly improve performance of the stock market forecast tools.

* The DE based evolutionary optimization of hyper-parameter codes of GpOls agents can autonomously yield evolutionary optimal forecast models without a need for expert knowledge on the algorithms and data content. Consequently, an active stock market index forecaster system with the DEHypGpOls algorithm can be implemented to the data-driven investment recommendations.

\section{Statements and Declarations}

Funding This study didn't receive any funding.

Author contributions All authors contributed to the study conception and design. The analysis and code were done by DA and BBA. The first draft of the manuscript was written and then polished by DA and BBA. All authors read and approved the final manuscript.

Conflict of interest The authors declare that they have no conflict of interest.

Ethical approval This article does not contain any studies with human participants or animals performed by any of the authors. 
Informed consent Informed consent was obtained from all individual participants included in the study.

\section{References}

Akbilgic O, Bozdogan H, Balaban ME (2014) A novel Hybrid RBF Neural Networks model as a forecaster. Statistics and Computing 24:365-375. https://doi.org/10.1007/s11222-013-9375-7

Ananthi M, Vijayakumar K (2020) Stock market analysis using candlestick regression and market trend prediction (CKRM). Journal of Ambient Intelligence and Humanized Computing 12:48194826. https://doi.org/10.1007/s12652-020-01892-5

Ari D, Alagoz BB (2021) A Review of Genetic Programming Popular Techniques, Fundamental Aspects, Software Tools and Applications. Sakarya University Journal of Science. https://doi.org/10.16984/saufenbilder.793333

Ashofteh P-S, Haddad OB, Loáiciga HA (2015) Evaluation of Climatic-Change Impacts on Multiobjective Reservoir Operation with Multiobjective Genetic Programming. Journal of Water Resources Planning and Management 141:04015030. https://doi.org/10.1061/(ASCE)WR.19435452.0000540

Bagheri A, Mohammadi Peyhani H, Akbari M (2014) Financial forecasting using ANFIS networks with Quantum-behaved Particle Swarm Optimization. Expert Systems with Applications 41:6235-6250. https://doi.org/10.1016/j.eswa.2014.04.003

Bisoi R, Dash PK (2014) A hybrid evolutionary dynamic neural network for stock market trend analysis and prediction using unscented Kalman filter. Applied Soft Computing Journal 19:4156. https://doi.org/10.1016/j.asoc.2014.01.039

Burke E, Kendall G, Newall J, et al (2003) Hyper-Heuristics: An Emerging Direction in Modern Search Technology. In: Handbook of Metaheuristics. Kluwer Academic Publishers, Boston, pp 457-474

Castelli M, Trujillo L, Vanneschi L, Popovič A (2015) Prediction of energy performance of residential buildings: A genetic programming approach. Energy and Buildings 102:67-74. https://doi.org/10.1016/j.enbuild.2015.05.013

Chatzimparmpas A, Martins RM, Kucher K, Kerren A (2021) VisEvol: Visual Analytics to Support Hyperparameter Search through Evolutionary Optimization. Computer Graphics Forum 40:201214. https://doi.org/10.1111/cgf.14300

Chen W, Jiang M, Zhang WG, Chen Z (2021) A novel graph convolutional feature based convolutional neural network for stock trend prediction. Information Sciences 556:67-94. https://doi.org/10.1016/j.ins.2020.12.068

Cho H, Kim Y, Lee E, et al (2020) Basic Enhancement Strategies When Using Bayesian Optimization for Hyperparameter Tuning of Deep Neural Networks. IEEE Access 8:5258852608. https://doi.org/10.1109/ACCESS.2020.2981072

Cui L, Li G, Zhu Z, et al (2018) A novel differential evolution algorithm with a self-adaptation parameter control method by differential evolution. Soft Computing 22:6171-6190. https://doi.org/10.1007/s00500-017-2685-5

Dal Piccol Sotto LF, De Melo VV (2014) Investigation of linear genetic programming techniques for symbolic regression. Proceedings - 2014 Brazilian Conference on Intelligent Systems, BRACIS 2014 146-151. https://doi.org/10.1109/BRACIS.2014.36

De Falco I, Della Cioppa A, Koutny T, et al (2018) Genetic Programming-based induction of a glucose-dynamics model for telemedicine. Journal of Network and Computer Applications 
Fallahpour A, Olugu EU, Musa SN, et al (2016) An integrated model for green supplier selection under fuzzy environment: application of data envelopment analysis and genetic programming approach. Neural Computing and Applications 27:707-725. https://doi.org/10.1007/s00521-0151890-3

Feli M, Abdali-Mohammadi F (2019) A novel recursive backtracking genetic programming-based algorithm for 12-lead ECG compression. Signal, Image and Video Processing 13:1029-1036. https://doi.org/10.1007/s11760-019-01441-4

Feurer M, Hutter F (2019) Hyperparameter Optimization. In: Hutter F., Kotthoff L., Vanschoren J. (eds) Automated Machine Learning. The Springer Series on Challenges in Machine Learning. Springer, pp 3-33

Gandhmal DP, Kumar K (2019) Systematic analysis and review of stock market prediction techniques. Computer Science Review 34:100190. https://doi.org/10.1016/j.cosrev.2019.08.001

Garcia-Almanza AL, Tsang EPK (2006) Forecasting stock prices using Genetic Programming and Chance Discovery. 12th International Conference On Computing In Economics And Finance number 489

García CE, Prett DM, Morari M (1989) Model predictive control: Theory and practice-A survey. Automatica 25:335-348. https://doi.org/10.1016/0005-1098(89)90002-2

Gideon J, Khorram S, Aldeneh Z, et al (2017) Progressive Neural Networks for Transfer Learning in Emotion Recognition

Hiransha M, Gopalakrishnan EA, Menon VK, Soman KP (2018) NSE Stock Market Prediction Using Deep-Learning Models. Procedia Computer Science 132:1351-1362. https://doi.org/10.1016/j.procs.2018.05.050

Hsu CM (2011) A hybrid procedure for stock price prediction by integrating self-organizing map and genetic programming. Expert Systems with Applications 38:14026-14036. https://doi.org/10.1016/j.eswa.2011.04.210

Huang C-F (2012) A hybrid stock selection model using genetic algorithms and support vector regression. Applied Soft Computing 12:807-818. https://doi.org/10.1016/j.asoc.2011.10.009

Ingle V, Deshmukh S (2021) Ensemble deep learning framework for stock market data prediction (EDLF-DP). Global Transitions Proceedings 2:47-66. https://doi.org/10.1016/j.gltp.2021.01.008

Kaboli SHA, Fallahpour A, Selvaraj J, Rahim NA (2017) Long-term electrical energy consumption formulating and forecasting via optimized gene expression programming. Energy 126:144-164. https://doi.org/10.1016/j.energy.2017.03.009

Karatahansopoulos A, Sermpinis G, Laws J, Dunis C (2014) Modelling and Trading the Greek Stock Market with Gene Expression and Genetic Programing Algorithms. Journal of Forecasting 33:596-610. https://doi.org/10.1002/for.2290

Keshavarz A, Mehramiri M (2015) New Gene Expression Programming models for normalized shear modulus and damping ratio of sands. Engineering Applications of Artificial Intelligence 45:464472. https://doi.org/10.1016/j.engappai.2015.07.022

Koza JR (1992) Genetic programming: on the programming of computers by means of natural selection, MIT press.

Koza JR, Keane MA, Streeter MJ (2004) Routine automated synthesis of five patented analog circuits using genetic programming. Soft Computing 8:318-324. https://doi.org/10.1007/s00500-003- 
0288-9

Kumar Chandar S (2021) Grey Wolf optimization-Elman neural network model for stock price prediction. Soft Computing 25:649-658. https://doi.org/10.1007/s00500-020-05174-2

Kuo CS, Hong TP, Chen CL (2007) Applying genetic programming technique in classification trees. Soft Computing 11:1165-1172. https://doi.org/10.1007/s00500-007-0159-X

Kwon W, Bruckstein A, Kailath T (1982) Stabilizing state-feedback design via the moving horizon method. In: 1982 21st IEEE Conference on Decision and Control. IEEE, pp 234-239

Li H, Wong ML (2015) Financial Fraud Detection by using Grammar-based Multi-objective Genetic Programming with ensemble learning. 2015 IEEE Congress on Evolutionary Computation, CEC 2015 - Proceedings 1113-1120. https://doi.org/10.1109/CEC.2015.7257014

Liang J, Wen J, Wang Z, Wang J (2020) Evolving semantic object segmentation methods automatically by genetic programming from images and image processing operators. Soft Computing 24:12887-12900. https://doi.org/10.1007/s00500-020-04713-1

Liashchynskyi P, Liashchynskyi P (2019) Grid Search, Random Search, Genetic Algorithm: A Big Comparison for NAS

Liu L, Shao L, Li X, Lu K (2016) Learning Spatio-Temporal Representations for Action Recognition: A Genetic Programming Approach. IEEE Transactions on Cybernetics 46:158-170. https://doi.org/10.1109/TCYB.2015.2399172

Lo AW, MacKinlay AC (1988) Stock Market Prices Do Not Follow Random Walks: Evidence from a Simple Specification Test. A Non-Random Walk Down Wall Street 17-46. https://doi.org/10.1515/9781400829095.17

López-López VR, Trujillo L, Legrand P (2019) Applying genetic improvement to a genetic programming library in C++. Soft Computing 23:11593-11609. https://doi.org/10.1007/s00500018-03705-6

Madár J, Abonyi J, Szeifert F (2005) Genetic Programming for the Identification of Nonlinear Input-Output Models. Industrial \& Engineering Chemistry Research 44:3178-3186. https://doi.org/10.1021/ie049626e

Mahmud MS, Meesad P (2016) An innovative recurrent error-based neuro-fuzzy system with momentum for stock price prediction. Soft Computing 20:4173-4191. https://doi.org/10.1007/s00500-015-1752-z

Markose S, Tsang E, Er H, Salhi A (2001) Evolutionary arbitrage for FTSE-100 index options and futures. In: Proceedings of the 2001 Congress on Evolutionary Computation (IEEE Cat. No.01TH8546). IEEE, pp 275-282

Moghar A, Hamiche M (2020) Stock Market Prediction Using LSTM Recurrent Neural Network. Procedia Computer Science 170:1168-1173. https://doi.org/10.1016/j.procs.2020.03.049

Mora J, Salvador R, de la Torre E (2019) On the scalability of evolvable hardware architectures: comparison of systolic array and Cartesian genetic programming. Springer US

Nguyen S, Mei Y, Zhang M (2017) Genetic programming for production scheduling: a survey with a unified framework. Complex \& Intelligent Systems 3:41-66. https://doi.org/10.1007/s40747017-0036-X

Ollar J, Mortished C, Jones R, et al (2017) Gradient based hyper-parameter optimisation for well conditioned kriging metamodels. Structural and Multidisciplinary Optimization 55:2029-2044. https://doi.org/10.1007/s00158-016-1626-8 
Pang X, Zhou Y, Wang P, et al (2020) An innovative neural network approach for stock market prediction. The Journal of Supercomputing 76:2098-2118. https://doi.org/10.1007/s11227-0172228-y

Patnaik AK, Bhuyan PK (2016) Application of genetic programming clustering in defining LOS criteria of urban street in Indian context. Travel Behaviour and Society 3:38-50. https://doi.org/10.1016/j.tbs.2015.08.003

Qin AK, Huang VL, Suganthan PN (2009) Differential Evolution Algorithm With Strategy Adaptation for Global Numerical Optimization. IEEE Transactions on Evolutionary Computation 13:398-417. https://doi.org/10.1109/TEVC.2008.927706

Rajab S, Sharma V (2019) An interpretable neuro-fuzzy approach to stock price forecasting. Soft Computing 23:921-936. https://doi.org/10.1007/s00500-017-2800-7

Schmidt M, Safarani S, Gastinger J, et al (2019) On the Performance of Differential Evolution for Hyperparameter Tuning. In: 2019 International Joint Conference on Neural Networks (IJCNN). IEEE, pp 1-8

Shao L, Liu L, Li X (2014) Feature learning for image classification via multiobjective genetic programming. IEEE Transactions on Neural Networks and Learning Systems 25:1359-1371. https://doi.org/10.1109/TNNLS.2013.2293418

Shen J, Shafiq MO (2020) Short-term stock market price trend prediction using a comprehensive deep learning system. Journal of Big Data 7:. https://doi.org/10.1186/s40537-020-00333-6

Sheta A, Faris H, Alkasassbeh M (2013) A Genetic Programming Model for S\&P 500 Stock Market Prediction. International Journal of Control and Automation 6:303-314. https://doi.org/10.14257/ijca.2013.6.6.29

Shoaib M, Shamseldin AY, Melville BW, Khan MM (2015) Runoff forecasting using hybrid Wavelet Gene Expression Programming (WGEP) approach. Journal of Hydrology 527:326-344. https://doi.org/10.1016/j.jhydrol.2015.04.072

Shu-Heng Chen (2002) Genetic Algorithms and Genetic Programming in Computational Finance. Springer US, Boston, MA

Sikulova M, Komjathy G, Sekanina L (2014) Towards compositional coevolution in evolutionary circuit design. IEEE SSCI 2014 - 2014 IEEE Symposium Series on Computational Intelligence IEEE ICES: 2014 IEEE International Conference on Evolvable Systems, Proceedings 157-164. https://doi.org/10.1109/ICES.2014.7008735

Solomatine D, See LM, Abrahart RJ (2009) Data-Driven Modelling: Concepts, Approaches and Experiences. In: Practical Hydroinformatics. Springer Berlin Heidelberg, Berlin, Heidelberg, pp $17-30$

Storn R, Price K (1995) Differential evolution - A simple and efficient adaptive scheme for global optimization over continuous spaces. Journal of Global Optimization 1-12

Storn R, Price K (1997) Differential Evolution - A Simple and Efficient Heuristic for Global Optimization over Continuous Spaces. Journal of Global Optimization 341:284-287

Subhi Alzazah F, Cheng X (2020) Recent Advances in Stock Market Prediction Using Text Mining: A Survey. In: E-Business - Higher Education and Intelligence Applications. IntechOpen

Suganuma M, Shirakawa S, Nagao T (2017) A genetic programming approach to designing convolutional neural network architectures. In: Proceedings of the Genetic and Evolutionary Computation Conference on - GECCO '17. ACM Press, New York, New York, USA, pp 497504 
Tahmassebi A, Gandomi AH (2018) Building energy consumption forecast using multi-objective genetic programming. Measurement 118:164-171. https://doi.org/10.1016/j.measurement.2018.01.032

Thakkar A, Chaudhari K (2021) A Comprehensive Survey on Portfolio Optimization, Stock Price and Trend Prediction Using Particle Swarm Optimization. Springer Netherlands

Tran B, Xue B, Zhang M (2016) Genetic programming for feature construction and selection in classification on high-dimensional data. Memetic Computing 8:3-15. https://doi.org/10.1007/s12293-015-0173-y

Trierweiler Ribeiro G, Alves Portela Santos A, Cocco Mariani V, dos Santos Coelho L (2021) Novel hybrid model based on echo state neural network applied to the prediction of stock price return volatility. Expert Systems with Applications 184:115490. https://doi.org/10.1016/j.eswa.2021.115490

Trujillo L, Álvarez González E, Galván E, et al (2020) On the analysis of hyper-parameter space for a genetic programming system with iterated F-Race. Soft Computing 24:14757-14770. https://doi.org/10.1007/s00500-020-04829-4

Villalobos-Arias L, Quesada-López C (2021) Comparative study of random search hyper-parameter tuning for software effort estimation. In: Proceedings of the 17th International Conference on Predictive Models and Data Analytics in Software Engineering. ACM, New York, NY, USA, pp $21-29$

Wang L, Wang Z, Zhao S, Tan S (2015) Stock market trend prediction using dynamical Bayesian factor graph. Expert Systems with Applications 42:6267-6275. https://doi.org/10.1016/j.eswa.2015.01.035

Wiese JJ (2011) System Identification and Model-Based Control of a Filter Cake Drying Process. University of Stellenbosch

Wu J, Chen XY, Zhang H, et al (2019) Hyperparameter optimization for machine learning models based on Bayesian optimization. Journal of Electronic Science and Technology 17:26-40. https://doi.org/10.11989/JEST.1674-862X.80904120

Yang F, Chen J, Liu Y (2021) Improved and optimized recurrent neural network based on PSO and its application in stock price prediction. Soft Computing 5:. https://doi.org/10.1007/s00500-02106113-5

Yoo PD, Kim MH, Jan T (2005) Machine learning techniques and use of event information for stock market prediction: A survey and evaluation. In: Proceedings - International Conference on Computational Intelligence for Modelling, Control and Automation, CIMCA 2005 and International Conference on Intelligent Agents, Web Technologies and Internet. IEEE, Vienna, Austria, pp 835-841

Zafra A, Ventura S (2012) Multi-instance genetic programming for predicting student performance in web based educational environments. Applied Soft Computing 12:2693-2706. https://doi.org/10.1016/j.asoc.2012.03.054

Zhuang F, Qi Z, Duan K, et al (2021) A Comprehensive Survey on Transfer Learning. Proceedings of the IEEE 109:43-76. https://doi.org/10.1109/JPROC.2020.3004555 